\title{
Erratum to: Parallel vertex approximate gradient discretization of hybrid dimensional Darcy flow and transport in discrete fracture networks
}

\author{
Feng Xing ${ }^{1,2} \cdot$ Roland Masson $^{1} \cdot$ Simon Lopez ${ }^{2}$
}

Published online: 6 June 2017

(c) Springer International Publishing Switzerland 2017

\section{Erratum to: Comput Geosci}

\section{DOI 10.1007/s10596-016-9606-z}

The original version of this article unfortunately contained mistakes in few displayed and inline equations. The occurrences of " $n$ " character were inadvertently deleted.

The corrected displayed and inline equations are presented below:

5th displayed equation, paragraph 10 under Discrete fracture network and functional setting

$$
\begin{aligned}
H(\Omega, \Gamma)= & \left\{\mathbf{q}_{m}=\left(\mathbf{q}_{m, \alpha}\right)_{\alpha \in \mathcal{A}}, \mathbf{q}_{f}=\left(\mathbf{q}_{f, i}\right)_{i \in I} \mid\right. \\
& \mathbf{q}_{m} \in H_{\operatorname{div}}(\Omega \backslash \bar{\Gamma}), \mathbf{q}_{f, i} \in L^{2}\left(\Gamma_{i}\right)^{d-1}, \\
& \left.\operatorname{div}_{\tau_{i}}\left(\mathbf{q}_{f, i}\right)-\gamma_{\mathbf{n}, i}^{+} \mathbf{q}_{m}-\gamma_{\mathbf{n}, i}^{-} \mathbf{q}_{m} \in L^{2}\left(\Gamma_{i}\right), i \in I\right\},
\end{aligned}
$$

The online version of the original article can be found at http://dx.doi.org/10.1007/s10596-016-9606-z.

Feng Xing

feng.xing@unice.fr

Roland Masson

roland.masson@unice.fr

Simon Lopez

s.lopez@brgm.fr

1 Laboratoire de Mathématiques J.A. Dieudonné, UMR 7351 CNRS, University Nice Sophia Antipolis, team COFFEE, INRIA Sophia Antipolis Méditerranée, Parc Valrose 06108 Nice Cedex 02, France

2 BRGM, Scientific and Technical Center, 3 Avenue Claude Guillemin, BP 36009, 45060 Orléans Cedex 2, France
Equation 1

$$
\begin{aligned}
W(\Omega, \Gamma)= & \left\{\left(\mathbf{q}_{m}, \mathbf{q}_{f}\right) \in H(\Omega, \Gamma) \mid \sum_{\alpha \in \mathcal{A}} \int_{\Omega_{\alpha}}\left(\mathbf{q}_{m, \alpha} \cdot \nabla v\right.\right. \\
& \left.+\operatorname{div}\left(\mathbf{q}_{m, \alpha}\right) v\right) d \mathbf{x}+\sum_{i \in I} \int_{\Gamma_{i}}\left(\mathbf{q}_{f, i} \cdot \nabla_{\tau_{i}} \gamma_{i} v\right. \\
& \left.+\left(\operatorname{div}_{\tau_{i}}\left(\mathbf{q}_{f, i}\right)-\gamma_{\mathbf{n}, i}^{+} \mathbf{q}_{m}-\gamma_{\mathbf{n}, i}^{-} \mathbf{q}_{m}\right) \gamma_{i} v\right) d \tau(\mathbf{x}) \\
& \left.=0 \forall v \in V^{0}\right\} .
\end{aligned}
$$

2nd, 3rd and 5th sentence, paragraph 11 under Discrete fracture network and functional setting

The last definition corresponds to imposing in a weak sense the conditions $\sum_{i \in I} \gamma_{\mathbf{n}, \Sigma_{i}} \mathbf{q}_{f, i}=0$ on $\Sigma \backslash \Sigma_{0}$ and $\gamma_{\mathbf{n}, \Sigma_{i}} \mathbf{q}_{f, i}=0$ on $\Sigma_{i, N}, i \in I$, where $\gamma_{\mathbf{n}, \Sigma_{i}}$ is the normal trace operator on $\Sigma_{i}$ (tangent to $\Gamma_{i}$ ) with the normal oriented outward from $\Gamma_{i}$, and using the extension of $\gamma_{\mathbf{n}, \Sigma_{i}} \mathbf{q}_{f, i}$ by zero on $\Sigma \backslash \Sigma_{i}$.

Equation 2

$$
\left\{\begin{aligned}
\operatorname{div}\left(\mathbf{q}_{m, \alpha}\right) & =0 & & \text { on } \Omega_{\alpha}, \alpha \in \mathcal{A}, \\
\mathbf{q}_{m, \alpha} & =-\frac{\Lambda_{m}}{\mu} \nabla u & & \text { on } \Omega_{\alpha}, \alpha \in \mathcal{A}, \\
\operatorname{div}_{\tau_{i}}\left(\mathbf{q}_{f, i}\right)-\gamma_{\mathbf{n}, i}^{+} \mathbf{q}_{m}-\gamma_{\mathbf{n}, i}^{-} \mathbf{q}_{m} & =0 & & \text { on } \Gamma_{i}, i \in I, \\
\mathbf{q}_{f, i} & =-d_{f} \frac{\Lambda_{f}}{\mu} \nabla_{\tau_{i}} \gamma_{i} u & & \text { on } \Gamma_{i}, i \in I .
\end{aligned}\right.
$$


3rd sentence, paragraph 1 under Hybrid dimensional transport model

Let $\gamma_{\mathbf{n}}$ be the normal trace operator on $\partial \Omega$ with the normal oriented outward from $\Omega$. Let us define $\partial \Omega^{-}=\{\mathbf{x} \in$ $\left.\partial \Omega \mid \gamma_{\mathbf{n}} \mathbf{q}_{m}(\mathbf{x})<0\right\}, \Sigma_{i, 0}^{-}=\left\{\mathbf{x} \in \Sigma_{i, 0} \mid \gamma_{\mathbf{n}, \Sigma_{i}} \mathbf{q}_{f, i}(\mathbf{x})<\right.$ $0\}, i \in I$, as well as the following subset of $\Sigma \backslash \Sigma_{0}$ :

$$
\begin{aligned}
\phi_{m} \partial_{t} c_{m, \alpha}+\operatorname{div}\left(c_{m, \alpha} \mathbf{q}_{m, \alpha}\right) & =0 \\
\phi_{f} d_{f} \partial_{t} c_{f, i}+\operatorname{div}_{\tau_{i}}\left(c_{f, i} \mathbf{q}_{f, i}\right) & =\gamma_{\mathbf{n}, i}^{+} c_{m} \mathbf{q}_{m}+\gamma_{\mathbf{n}, i}^{-} c_{m} \mathbf{q}_{m} \\
\left(\gamma_{\mathbf{n}, i}^{ \pm} c_{m} \mathbf{q}_{m}\right)^{-} & =c_{f}\left(\gamma_{\mathbf{n}, i}^{ \pm} \mathbf{q}_{m}\right)^{-} \\
\left(\gamma_{\mathbf{n}, \Sigma_{i}} c_{f, i} \mathbf{q}_{f, i}\right)^{-} & =c_{f, \Sigma^{-}}\left(\gamma_{\mathbf{n}, \Sigma_{i}} \mathbf{q}_{f, i}\right)^{-} \\
\sum_{j \in I} \gamma_{\mathbf{n}, \Sigma_{j}} c_{f, j} \mathbf{q}_{f, j} & =0 \\
\left(\gamma_{\mathbf{n}} c_{m} \mathbf{q}_{m}\right)^{-} & =\bar{c}_{m}\left(\gamma_{\mathbf{n}} q_{m}\right)^{-} \\
\left(\gamma_{\mathbf{n}, \Sigma_{i}} c_{f, i} \mathbf{q}_{f, i}\right)^{-} & =\bar{c}_{f, i}\left(\gamma_{\mathbf{n}, \Sigma_{i}} \mathbf{q}_{f, i}\right)^{-} \\
c_{m} & =c_{m}^{0} \\
c_{f} & =c_{f}^{0}
\end{aligned}
$$

1st displayed equation, paragraph 1 under Hybrid dimensional transport model

$$
\Sigma^{-}=\left\{\mathbf{x} \in \Sigma \backslash \Sigma_{0}\left|\sum_{i \in I}\right| \gamma_{\mathbf{n}, \Sigma_{i}} \mathbf{q}_{f, i}(\mathbf{x}) \mid \neq 0\right\} .
$$

Equation 4

on $\Omega_{\alpha} \times(0, T), \alpha \in \mathcal{A}$

on $\Gamma_{i} \times(0, T), i \in I$,

on $\Gamma_{i} \times(0, T), i \in I$,

on $\left(\Sigma_{i} \backslash \Sigma_{i, 0}\right) \times(0, T), i \in I$,

on $\left(\Sigma \backslash \Sigma_{0}\right) \times(0, T)$,

on $\partial \Omega \times(0, T)$,

on $\Sigma_{i, 0} \times(0, T), i \in I$,

on $(\Omega \backslash \bar{\Gamma}) \times\{t=0\}$,

on $\Gamma \times\{t=0\}$, 